\title{
Grounding co-occurrence: Identifying features in a lexical co-occurrence model of semantic memory
}

\author{
KeVIn Durda, LoRi Buchanan, ANd RichaRd Caron \\ University of Windsor, Windsor, Ontario, Canada
}

\begin{abstract}
Lexical co-occurrence models of semantic memory represent word meaning by vectors in a high-dimensional space. These vectors are derived from word usage, as found in a large corpus of written text. Typically, these models are fully automated, an advantage over models that represent semantics that are based on human judgments (e.g., feature-based models). A common criticism of co-occurrence models is that the representations are not grounded: Concepts exist only relative to each other in the space produced by the model. It has been claimed that feature-based models offer an advantage in this regard. In this article, we take a step toward grounding a cooccurrence model. A feed-forward neural network is trained using back propagation to provide a mapping from co-occurrence vectors to feature norms collected from subjects. We show that this network is able to retrieve the features of a concept from its co-occurrence vector with high accuracy and is able to generalize this ability to produce an appropriate list of features from the co-occurrence vector of a novel concept.
\end{abstract}

The organizational structure of semantic knowledge remains an open and difficult problem in psycholinguistics. Early researchers proposed a hierarchical structure (Collins \& Quillian, 1969) in which concepts are represented as localist nodes arranged by means of category inclusion. General concepts (e.g., living-thing) are stored near the top of the hierarchy, and more specific concepts are stored lower in the tree. For example, sparrow is stored as a subordinate of the category bird, which is, in turn, stored as a subordinate of animal, and so on. Attributes possessed by each concept are attached to the hierarchy at the shallowest possible level by means of links that represent the different types of relationships between concepts. These properties are inherited by all of the subordinate concepts. Later revisions of this model place less emphasis on the structure of semantic information and focus on the mechanics of semantic processing.

In one revision of the Collins and Quillian (1969) model, Collins and Loftus (1975) proposed a spreading activation network. Concepts are again represented as nodes that are connected by various types of relational links; however, the categorical structure of the network was abandoned in favor of a more general structure having no explicit hierarchy. Categorical information is preserved in the network by a set of IS-A links (e.g., a sparrow IS-A bird). Processing of a concept produces activation of that concept in the network, which spreads to related concepts via the relational links. This spread of activation facilitates later processing of related concepts. Although these models can accommodate a large array of empirical phenomena, there are many shortcomings (Rogers \& McClelland, 2004, chap. 1). In particular, the category inclusion relation represents only taxonomically organized categories, and even then only for those exemplars that are most typical of the category (Sloman, 1998; Steyvers \& Tenenbaum, 2005). Additionally, there is no mechanism described by which semantic networks can be constructed (Rogers \& McClelland, 2004). Steyvers and Tenenbaum (2005) and Lemaire and Denhiére (2004) have proposed algorithms that construct basic semantic networks, but the resulting networks do not approach the richness of human semantics.

Distributed representations (see, e.g., Hinton, McClelland, \& Rumelhart, 1986) offer an alternative to the representational scheme used above. In contrast to a localist representation, where a single dedicated node is assigned to each concept, a distributed scheme represents a concept as a unique pattern of activation across a common set of nodes. This method of representation has found support in many studies (as surveyed in Saffran, 2000). The double dissociations in reading performance evidenced by individuals with semantic dementia or brain injury suggest that words are not represented together in a single region of the brain, but rather are distributed throughout the cortex. At a larger scale, distributed processing is an integral aspect of theories of grounded (or embodied) cognition that posit that all knowledge is represented across different modalities and that language is closely tied to other cognitive systems, such as the perceptual and motor systems (e.g., Barsalou, 2008). 
Neural network and connectionist models of cognition commonly adopt distributed representations as the primary means of knowledge representation. Binary feature vectors have been used to represent semantic knowledge in a distributed manner in many models (Harm \& Seidenberg, 2004; Hinton \& Shallice, 1991; McRae, de Sa, \& Seidenberg, 1997; Plaut \& Shallice, 1993; Rogers \& McClelland, 2004). Each component of the vector corresponds to a feature of a concept, such as $<$ has_wings $>$ or $<$ is_small $>$, and a concept is represented by activating all of the appropriate features possessed by the concept.

In one version of the distributed computational approach, McRae et al. (1997) developed feature vector representations based on a large number of associations produced by subjects and used these vectors to construct a semantic processing unit in a model that simulates access of semantic information from orthographic input. That work gained real-world relevance through the McRae, Cree, Seidenberg, and McNorgan (2005) norming for 541 concepts from 725 human subjects. This time-consuming norming is frequently bypassed in favor of an easier and more pragmatic, albeit arguably less psychologically valid, hand selection of features that are presumed to accurately represent the underlying semantics of the concepts (Hinton \& Shallice, 1991; Plaut \& Shallice, 1993) or they are generated automatically from other data sources; for instance, Harm and Seidenberg (2004) generated feature vectors from the WordNet database (Fellbaum, 1998). Other researchers (Plaut, 1995; Plaut \& Booth, 2000; Rodd, Gaskell, \& Marslen-Wilson, 2004) have relied on similarity between randomly generated binary vector representations. This is the least satisfying approach because the components of randomly generated vectors do not correspond meaningfully to properties of the underlying concepts.

Lexical co-occurrence models (Andrews, Vigliocco, \& Vinson, 2005, 2007, 2009; Burgess \& Lund, 2000; Griffiths \& Steyvers, 2002, 2003; Griffiths, Steyvers, \& Tenenbaum, 2007; Jones \& Mewhort, 2007; Landauer \& Dumais, 1997; Lund \& Burgess, 1996; Shaoul \& Westbury, 2006) offer an alternative method of constructing rich distributed semantic representations. These representations take the form of high-dimensional vectors constructed through analysis of word usage in a large corpus of written text. These "semantic space" models provide a much more convenient method of developing word meaning than their feature-based counterparts do: Meaning is derived directly and automatically from analyzing distributional information available in written text. This presents a great advantage in light of the amount of work required to acquire reasonably reliable feature norms from subjects. After a set of representational vectors is constructed, word similarity can be measured by the cosine or correlation between vector representations, or distance between concepts can be measured by any of various distance metrics, such as the city-block or Euclidean metrics. In contrast to the feature vectors described above, the individual components of these vectors do not necessarily align with properties of the concepts that they represent: Word meaning is represented in a distributed manner over all components of the vector.
Lexical co-occurrence models have been successful at modeling a number of empirical results from the psycholinguistic literature. The vectors produced by these models have been shown to contain categorical and grammatical information (Burgess, 1998; Burgess \& Lund, 1997a, 2000); predict behavioral data from semantic priming experiments (Burgess \& Lund, 1997b; Jones, Kintsch, \& Mewhort, 2006; Lund \& Burgess, 1996); capture elements of grammatical, morphological, and semantic ambiguity (Burgess, 2001; Burgess \& Lund, 1997a); and perform similarly to humans in tasks such as similarity judgment and synonym selection (Landauer \& Dumais, 1997). The representations produced by lexical co-occurrence models are similar to those produced by simple recurrent networks (Elman, 1990) that develop internal representations that are based on information in the temporal structure of language (Burgess \& Lund, 2000).

Despite many demonstrations that co-occurrence vectors capture aspects of word meaning, co-occurrence models have not been immune to criticism. A particularly troublesome criticism is that the representations constructed by these models are not grounded in the physical world (French \& Labiouse, 2002; Glenberg, 1997; Glenberg \& Robertson, 2000). That is, word meaning is represented only by a high-dimensional vector, often in a space with arbitrary dimensions (Jones \& Mewhort, 2007; Landauer \& Dumais, 1997; Schütze, 1992, 1998), and can be interpreted only in relation to other vectors residing in the same semantic space. This poses a problem for co-occurrence models because features and featural similarity have been shown to affect word recognition and are thought to play a role in the basic organization of semantic memory (Martin \& Chao, 2001; McRae, Cree, Westmacott, \& de Sa, 1999; McRae et al., 1997; Pexman, Holyk, \& Monfils, 2003; Sitnikova, West, Kuperberg, \& Holcomb, 2006; Vigliocco et al., 2006), and there are numerous studies that provide a case for the role of embodied representations in language processing (for a review, see Barsalou, 2008).

Burgess (1998, 2000; Burgess \& Lund, 2000) argued that the representations produced by some co-occurrence models are, by dint of their construction, grounded. In the hyperspace analogue to language (HAL; Lund \& Burgess, 1996), each component of a word's co-occurrence vector measures that word's association to some other word from the corpus. Burgess postulated that this provides a form of grounding of concepts in the linguistic environment. In addition, because abstract and concrete concepts are acquired through the same mechanism and represented in the same way, abstract concepts are also grounded. However, this argument closely mirrors the argument that has been used against co-occurrence models - namely, that co-occurrence vectors can only be interpreted relative to each other. Suggesting that vectors are grounded only in the linguistic environment does not approach a solution to the problem that vectors are not grounded in the physical world. To resolve this problem, one must demonstrate that co-occurrence vectors contain information that can be reliably mapped onto perceptual properties of the concepts that they are said to represent. Furthermore, this argument applies only to models such as HAL, in which vec- 
tor components are directly associated with symbols from the model's learning environment. Some co-occurrence models, such as BEAGLE (Jones \& Mewhort, 2007), produce vectors in which the axes of the semantic space are arbitrary. In these models, there is no relationship between individual vector components and the learning environment.

This difficulty is also relevant to models such as LSA (Landauer \& Dumais, 1997) and COALS (Rohde, Gonnerman, \& Plaut, 2004), in which the dimension of a representation is reduced by using singular value decomposition. This form of dimension reduction does not reduce noise in the vectors, as does the dimension reduction used in HAL, but is a process vital to knowledge acquisition. The components of the resulting vectors no longer correspond to symbols from the learning environment and, as is usually the case for distributed representations, cannot be interpreted individually. In addition to lower dimensional vectors, singular value decomposition provides a function that can be used to translate these vectors back to the original high-dimensional space, where Burgess's argument (described above) applies. However, in practice, only the reduced dimension vectors are used for calculating similarity between words, and it is important to show that these vectors can be grounded without the additional step of reproducing the high-dimensional vectors.

More practically, Shaoul and Westbury (2008a) attempted to extract features from a co-occurrence model and found very low precision in identifying featural relationships between concepts. This suggests that cooccurrence may not be an appropriate measure of this relationship.

Other researchers have taken more reconciliatory approaches to the debate between embodied and symbolic representations. Louwerse (2007) argued that language is both symbolic and embodied and that the meanings of symbols (words) are reliant upon their relationships both to other symbols and to embodied experiences. Louwerse (2007) called this the symbol interdependency hypothesis, postulating that symbols can always be grounded, but that language comprehension operates largely on symbolic representations and the grounded representations of words are not necessarily accessed during comprehension. This theory also argues that symbolic representations are "built onto" embodied representations and that a great deal of word meaning can be gleaned from the distributional information present in language usage. LSA is offered as a model of the mechanisms that underlie symbol interdependency. Indeed, Louwerse, Cai, Hu, Ventura, and Jeuniaux (2006) demonstrated that LSA does contain information that has typically been purported to reflect embodied cognition. By applying multidimensional scaling, LSA was able to correctly order (1) units of time by relative size, (2) the days of the week, and (3) the months of the year, as well as to identify geographical relationships between major U.S. cities and between major cities from around the globe. Evidence from studies of iconicity and word frequency suggests that embodied relationships influence the statistical structure of language to such an extent that the embodied relationships become encoded within the statistical structure (Louwerse, 2008).

Riordan and Jones (in press) compared representations derived from featural information to those derived from co-occurrence information in a variety of semantic clustering tasks. Co-occurrence models produced results comparable to those of feature-based models for both concrete nouns and action verbs, although the nature of the semantic information employed by each type of model was often very different. This suggests that, to a large extent, meaning is encoded redundantly in both featural and distributional information, and the relationship between these two sources of data is complementary. Andrews et al. (2005, $2007,2009)$ treated featural information and distributional information as joint distributions to be learned by a Bayesian model. They have demonstrated that the representations produced by this model are better able to reproduce behavioral data than are models that include only one of the two sources of information or treat the two data sources independently. Furthermore, Andrews et al. have posited that, as in the symbol interdependency hypothesis, only some concepts are grounded, and treating featural and cooccurrence information as joint distributions allows for chains of inference, during which experiential knowledge about a concept is generalized to other concepts. Howell, Jankowicz, and Becker (2005) used a simple recurrent network (Elman, 1990) augmented with noun-feature and verb-feature output units to learn the statistical structure of language. They tested the network's ability to predict the upcoming word in a stream of language. When feature information from subject norms was provided along with the linguistic input, the network's performance on the word prediction task was better than when randomized feature information was provided, indicating that sensorimotor information improves the network's ability to learn linguistic structure.

We assume that, consistent with the symbol interdependency hypothesis and the observations of Riordan and Jones (in press), embodied information is partially encoded within language structure; we present a model that attempts to identify this information and uses it to produce correct features of concepts from representations derived from language usage. In this model, co-occurrence vectors are derived from a large body of text and are then used to train a feed-forward back-propagation network (Rumelhart, Hinton, \& Williams, 1986) that maps from the vectors to a set of binary feature representations from the McRae et al. (2005) norms. We show that the network is able to recall, with very high accuracy, the featural characteristics of concepts on which the network is trained and that the network is able to generalize this ability to novel concepts. This method is offered as a partial solution to the symbol grounding problem. We demonstrate that there exists information within vectors derived from language usage that can readily be used to identify perceptual and functional properties of concepts, but we do not make any claim of how this featural information could be represented across different modalities in the brain or how these properties themselves can emerge. 


\section{A Co-Occurrence Model of Semantic Memory}

The co-occurrence model is a variation of the Windsor improved norms of distance and similarity of representations of semantics (WINDSORS; Durda \& Buchanan, 2008) model, which is similar to other models, such as HAL, LSA, and COALS. Semantic representations are derived from the number of co-occurrences between pairs of words. These counts are transformed in order to limit the effects of written frequency in the resulting representations, and the dimension of the representations is reduced using singular value decomposition. Word-word co-occurrence counts were collected from a subset of the University of Alberta newsgroup corpus (Shaoul \& Westbury, 2008b) containing all of the messages posted to over 47,000 Englishlanguage, nonbinary-file newsgroups between October 26, 2005, and December 29, 2005. The 49,483 most frequent tokens from the corpus that appear in the standard Unix dictionary but did not appear in a stop list of closed-class words were taken as the vocabulary of the model. All other tokens in the corpus were excluded from the co-occurrence counts and were replaced with a placeholder symbol to maintain the structure of the text. Capitalization and punctuation were ignored. In total, there were over 1.5 million unique messages and over 692 million tokens, of which nearly 593 million were counted as words.

Co-occurrence counts were collected by centering an 11-word window over each word of the corpus, in sequence. For each pair of words from the vocabulary $\left(w_{a}, w_{t}\right)$, the number of times that $w_{a}$ appeared in a window centered around an occurrence of $w_{t}$-denoted as $C\left(w_{a}, w_{t}\right)$-was counted and recorded in a co-occurrence matrix (the occurrence of $w_{t}$ with itself at the center of each window was not counted). This matrix contained one row and one column corresponding to each word in the model's vocabulary, with the column corresponding to the word at the center of the window. Note that if $w_{a}$ appeared multiple times in a single window centered on $w_{t}$, each instance of $w_{a}$ in the window was counted. For example, in the window [I slit the sheet the sheet I slit and on the] centered on the second occurrence of sheet, $C($ slit, sheet $)=2$; but $C($ sheet, sheet $)=1$ because the occurrence of sheet with itself at the center of the window is not counted. Some models have employed weighted co-occurrence counts, in which each position of the window is assigned a fixed weight that is used to scale cooccurrence counts before summing across window positions (Lund \& Burgess, 1996; Shaoul \& Westbury, 2006, 2008a). However, we assume that the sentence structure diversity in text from myriad producers makes any single set of weights impossible to derive, and we thus chose to use a uniformly weighted window.

Upon completion of the co-occurrence count collection, we reduced the effects of word frequency by using the log relative frequency ratio (Damerau, 1993). Word frequency is a well-known predictor of performance in many psycholinguistic tasks and may mask more subtle effects, such as true semantic effects. It is therefore important to determine the strength of the relationship between two words independently of their frequency in the particular corpus being analyzed. Indeed, Shaoul and Westbury
(2006) showed that word frequency varies greatly among corpora, particularly among low-frequency words. Of course, because each corpus acts as an analogue to an individual's linguistic experience, different sets of semantic vectors are produced, and these differences are likely to have some psychological reality. However, to the extent that we wish to generalize across corpora, there should remain strong similarities between the internal structures of semantic spaces constructed from them. Reducing the influence of word frequency is a step toward this generalization.

Given a pair of words $\left(w_{a}, w_{t}\right)$, we evaluate the importance of the number of occurrences of $w_{a}$ in a window around $w_{t}$, using the log relative frequency ratio given by $R\left(w_{a}, w_{t}\right)=\log \left[\mathrm{P}_{t}\left(w_{a}\right) / \mathrm{P}\left(w_{a}\right)\right]$, where $\mathrm{P}_{t}\left(w_{a}\right)$ is the probability that any of the words appearing in a window centered on an instance of $w_{t}$ is $w_{a}$, and where $\mathrm{P}\left(w_{a}\right)$ is the probability of finding $w_{a}$ independent of context. Positive values of $R\left(w_{a}, w_{t}\right)$ indicate that $w_{a}$ occurs more frequently in the presence of $w_{t}$ than it does independently of context; negative values indicate a lower than expected frequency of occurrence. The probabilities $\mathrm{P}_{t}\left(w_{a}\right)$ and $\mathrm{P}\left(w_{a}\right)$ are calculated from the frequency information collected from the corpus, with both the window size and corpus size taken into consideration. We have $\mathrm{P}_{t}\left(w_{a}\right)=C\left(w_{a}, w_{t}\right) /[(n-1)$. $\left.C\left(w_{t}\right)\right]$, where $C\left(w_{t}\right)$ is the number of times that $w_{t}$ appears in the corpus and $n$ is the window size. The probability of finding $w_{a}$ is given by $\mathrm{P}\left(w_{a}\right)=C\left(w_{a}\right) / N$, where $N$ is the number of words in the corpus.

Each entry in the word-word co-occurrence matrix is transformed using the log relative frequency ratio. To increase the sparseness of the matrix, all entries with an absolute log relative frequency ratio below 0.01 were set to zero. The resulting matrix contained about 67.4 million $(2.75 \%)$ nonzero entries.

Singular value decomposition was used to reduce the dimension of this transformed matrix from nearly 50,000 rows to only 300 . This resulted in much more compact representations than those provided by the raw co-occurrence matrix, and these more compact vectors are better suited to inclusion in a computationally demanding connectionist model.

Before proceeding, we will briefly demonstrate that the vectors constructed with this method have succeeded in capturing semantic information. We show that similarity between word vectors matches human performance in psycholinguistic experiments, and, with multidimensional scaling, we show that categorical discrimination is captured by the vectors.

To show that the similarities between word vectors are not influenced by word frequency, a random sample of 10,000 word pairs was selected from the model's vocabulary, and the similarity between the words in each pair was calculated. Similarity did not correlate with either the frequency of the first word in the pair $[r(9999)=-.014$, $p>.05]$ or the frequency of the second word $[r(9999)=$ $-.017, p>.05]$.

Multidimensional scaling has been used to demonstrate that categorical information is represented latently in cooccurrence vectors. Multidimensional scaling projects 
points from a high-dimensional space into a much lower dimensional space while preserving as best as possible the distance between each pair of points. Figure 1 shows the results of a 2-D scaling of concepts from categories of geographical locations, cities, body parts, and animals (Burgess \& Lund, 1997a). The words are clustered into groups corresponding to the four different categories. The similar categories of cities and geographical locations, although grouped in close regions of semantic space, still segregate into distinct collections of items. To verify that these groupings also exist in the original high-dimensional space and are not artifacts of the multidimensional scaling procedure, the similarity between each pair of words was calculated, and the within-group similarities were compared with the similarities between items from different groups. For each category, the similarity between each pair of items in the category was compared with the similarity of items from all of the other categories. Figure 2 shows the mean similarity between each pair of categories. Clearly, the similarity between pairs of words taken from the same category is higher than the similarity between pairs from different categories. This observation was verified using ANOVA. However, since the data are not independent, four separate ANOVAs were used, as opposed to a single analysis. These analyses showed that the animals were differentiated from the other three categories $[F(1,244)=134.17, p<.001]$, as were the body parts $[F(1,523)=577.35, p<.001]$, the cities
$[F(1,207)=159.42, p<.001]$, and the geographical locations $[F(1,277)=118.85, p<.001]$. An ANOVA comparing only the city and geographical location categories was conducted to determine whether the words from these two highly similar categories were differentiated in the high-dimensional space. This analysis showed that the cities were differentiated from the geographical locations $[F(1,75)=34.25, p<.001]$ and vice versa $[F(1,101)=$ $16.475, p<.001]$.

Next, we will show that the model can simulate subjects' performance in semantic priming tasks. Ferrand and New (2004) examined priming effects for associatively related (e.g., street-car) and semantically similar (e.g., street-road) pairs. They found an advantage for related versus unrelated pairs with both types of prime, with a larger priming effect for the semantically similar pairs. We simulated this priming experiment by calculating the cosine similarity between word vectors for the same set of word pairs used by Ferrand and New. An unrelated condition was simulated by randomly reassigning the primes to different targets, so that no primes were paired with their original targets. There was a main effect of relatedness $[F(1,153)=104.99, p<.001]$. The mean difference between the related and unrelated conditions for semantically related pairs was $0.268[t(34)=7.50, p<.001]$. For the associatively related pairs, this difference was 0.180 $[t(39)=6.78, p<.001]$. These results mimic those reported by Ferrand and New.

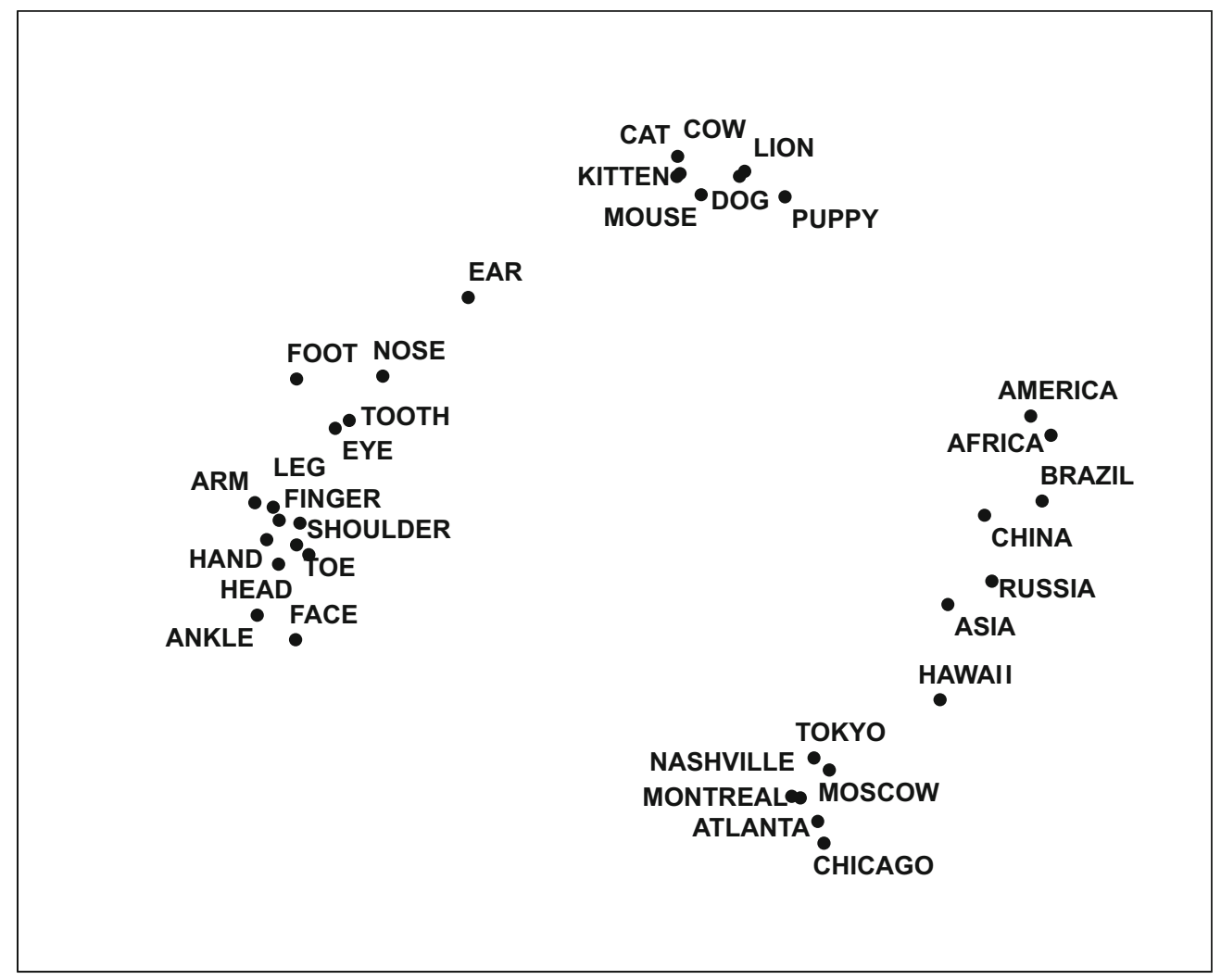

Figure 1. Two-dimensional scaling of concepts from categories of geographical locations, cities, body parts, and animals. 


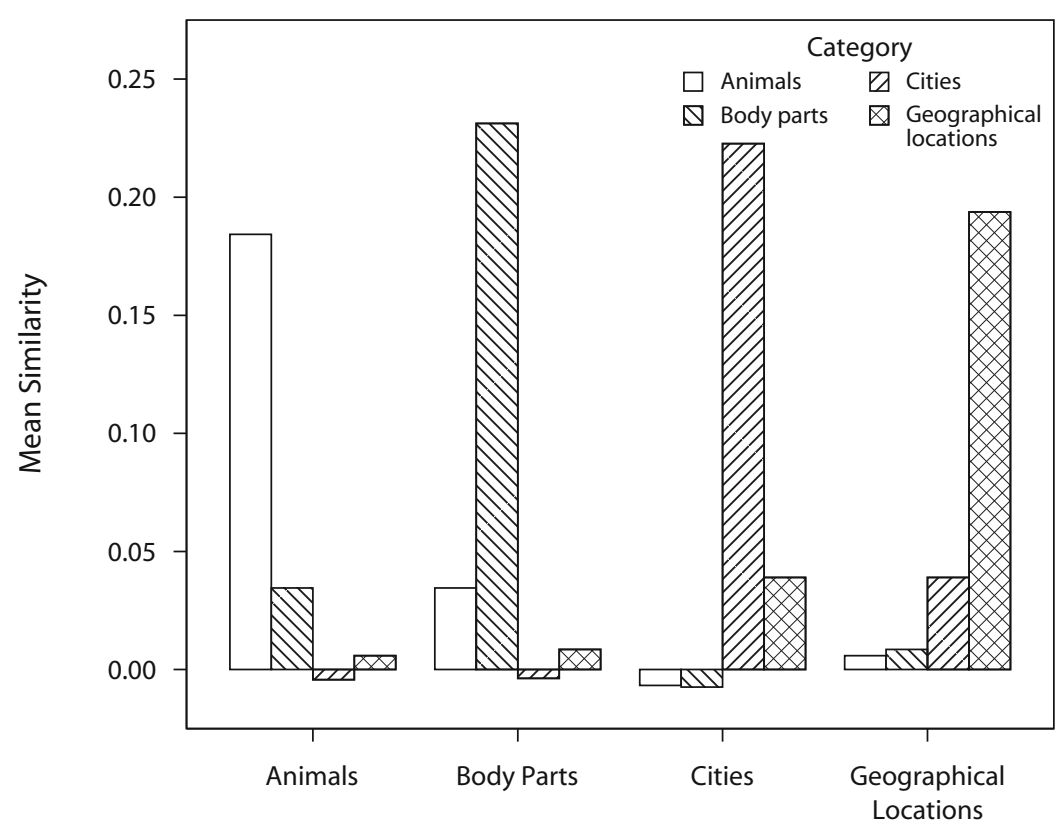

Category

Figure 2. Mean similarity between items in each pair of categories for four categories (animals, body parts, cities, geographical locations). Similarities between items from the same category are higher than those between items from different categories.

\section{A Neural Network Model of Feature Retrieval}

In this section, we will present a neural network model that learns to map from a concept's co-occurrence vector, as we described above, to a set of features that describe that concept. The network is a three-layer feed-forward network containing an input layer of 300 units, one layer of 583 hidden units, and an output layer containing 824 units, each denoting a feature that a concept may possess. Each unit in the input layer is connected to all hidden units, and each of the hidden units is connected to all output units. The inputs to the network are bipolar versions of the semantic vectors we described above. Input vectors are formed by setting all negative components of a concept's semantic vector to -1 and setting all other components to 1 .

The output patterns were derived from McRae et al.'s (2005) feature production norms. For each feature that was listed for two or more concepts, an associated output unit was included in the network. This resulted in a total of 824 output units, each representing a single feature. To create a concept's target representation, each output unit corresponding to a feature produced by one or more subjects for that concept was set to 1 , and all other output units were set to -1 . From the 541 words in the McRae et al. (2005) norms, only those that possessed 5 or more of the 824 features and appeared in the co-occurrence model's vocabulary were included. This resulted in a set of 465 words. From this set of words, we constructed 10 pairs of training and test sets. Each test set contained 20 of the 465 words, and the remaining 445 words were used for training. It is important to match the probability distribution of the features in each training-set and test-set pair, so the 20 items included in each test set could not simply be randomly selected. Instead, we used a genetic algorithm (Holland, 1992) to generate training sets with low Kullback-Leibler divergence (a measure of dissimilarity between probability distributions; Kullback \& Leibler, 1951) between the distribution of features in the training and test sets. Once the first test set was constructed, the words in it were excluded from consideration for the second test set, and the genetic algorithm was run again to produce a second test set of 20 concepts with low Kullback-Leibler divergence. This process was repeated until 10 test sets were generated, each time excluding the words from all previously generated test sets. In addition, from each of the 20 test patterns in each test set, a randomized test pattern was generated by randomly shuffling the components of the input vector but retaining the same target vector derived from the McRae et al. (2005) norms. These randomized patterns provided a control condition that will allow us to verify the network's ability to generalize to novel semantic representations. The network should identify features from the test set items accurately. However, for the randomized test vectors, which lack the structure of the original test items, we predict higher error rates and lower feature-recognition accuracy. The activations of the hidden and output units were calculated using a bipolar sigmoid function,

$$
f(x)=\frac{1-e^{-x}}{1+e^{-x}}
$$

where $x$ is the net input to the unit.

The network was trained using back propagation with momentum. In each epoch, every training pattern was pre- 
Table 1

Average Precision, Recall, and Accuracy for Training, Testing, and Randomized Test Patterns

\begin{tabular}{|c|c|c|c|c|c|c|}
\hline & \multicolumn{2}{|c|}{ Training } & \multicolumn{2}{|c|}{ Testing } & \multicolumn{2}{|c|}{$\begin{array}{c}\text { Randomized } \\
\text { Test }\end{array}$} \\
\hline & $M$ & $\overline{S D}$ & $M$ & $\overline{S D}$ & $M$ & $\overline{S D}$ \\
\hline Precision & 1.00 & .00 & .65 & .05 & .13 & .04 \\
\hline Recall & .97 & .00 & .23 & .03 & .04 & .01 \\
\hline$F$ & .99 & .00 & .34 & .03 & .06 & .02 \\
\hline
\end{tabular}

sented to the network, and weight changes were applied in a batch-updating procedure at the end of each epoch. The learning rate was set to 0.01 , and the momentum parameter was set to 0.9 .

\section{Results}

The performance of the model is evaluated by examining the average precision and recall over all training and test sets. Precision measures the proportion of correctly activated features in the list of all activated features. Recall measures the proportion of correctly activated features in the list of all features possessed by the concept. These measures can be combined into a single measure, the $F$ measure:

$$
F=\frac{2 \cdot \text { precision } \cdot \text { recall }}{\text { precision }+ \text { recall }} \text {. }
$$

Values of $F$ range from 0 to 1 , with higher values indicating better performance. The maximum value is attained when both precision and recall are perfect; the minimum is obtained when both precision and recall are 0 . We use precision, recall, and $F$ to measure network performance instead of accuracy (defined as the total proportion of output units that are correctly set, whether active or not, for a concept), because simply turning off all of the features for each concept results in an overall accuracy of over .98, due to the sparseness of the feature vectors.

Ten copies of the network were trained, each using a different training-set-test-set pair, for 3,500 epochs. At the end of training, the total sum squared error, averaged across training sets, was $608.73(S D=60.71)$. At the onset of training, the total error over all of the training patterns averaged over training set was 446,323.34 (SD = 7,939.97). There is still error remaining on the training patterns after training, but this error is drastically lower than that seen at the outset of training, and both precision and recall on the training sets were high. Mean precision, recall, and $F$ values for the training, testing, and randomized testing sets are shown in Table 1 . The average precision, recall, and $F$ throughout training for the training, testing, and randomized testing sets are shown in Figures 3, 4, and 5, respectively. A one-way ANOVA showed a main effect of item type (training, testing, randomized) on precision $[F(2,27)=1,164.60, p<.001]$, recall $[F(2,27)=8,776.32, p<.001]$, and $F[F(2,27)=$ $5,748.83, p<.001]$. Precision for training items was higher than precision on testing items $[t(9)=20.58, p<$ $.001]$ and on randomized items $[t(9)=61.23, p<.001]$. This pattern was repeated in the recall data, where training items had higher recall than that for test items $[t(9)=$ 87.26, $p<.001]$ and randomized items $[t(9)=211.64$, $p<.001]$. This pattern was also repeated in the $F$ data for test items $[t(9)=70.55, p<.001]$ and randomized items $[t(9)=148.94, p<.001]$. Performance was better on

\section{Training Data}

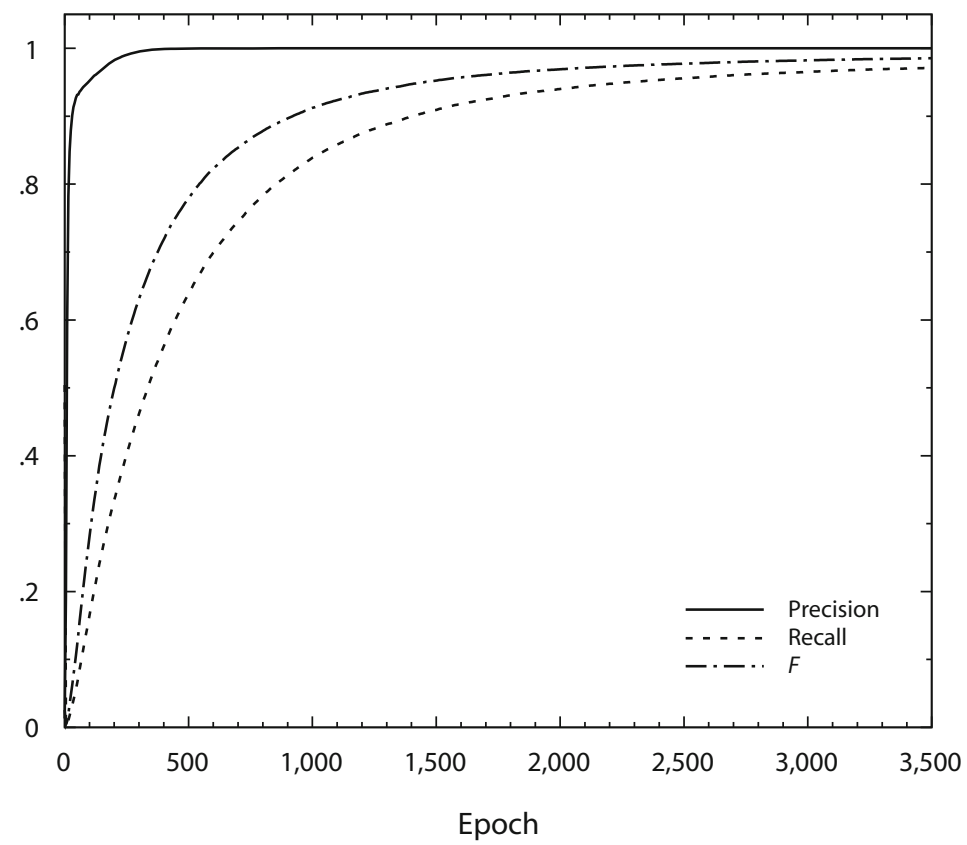

Figure 3. Average precision, recall, and $F$ for 465 training patterns. 
Testing Data

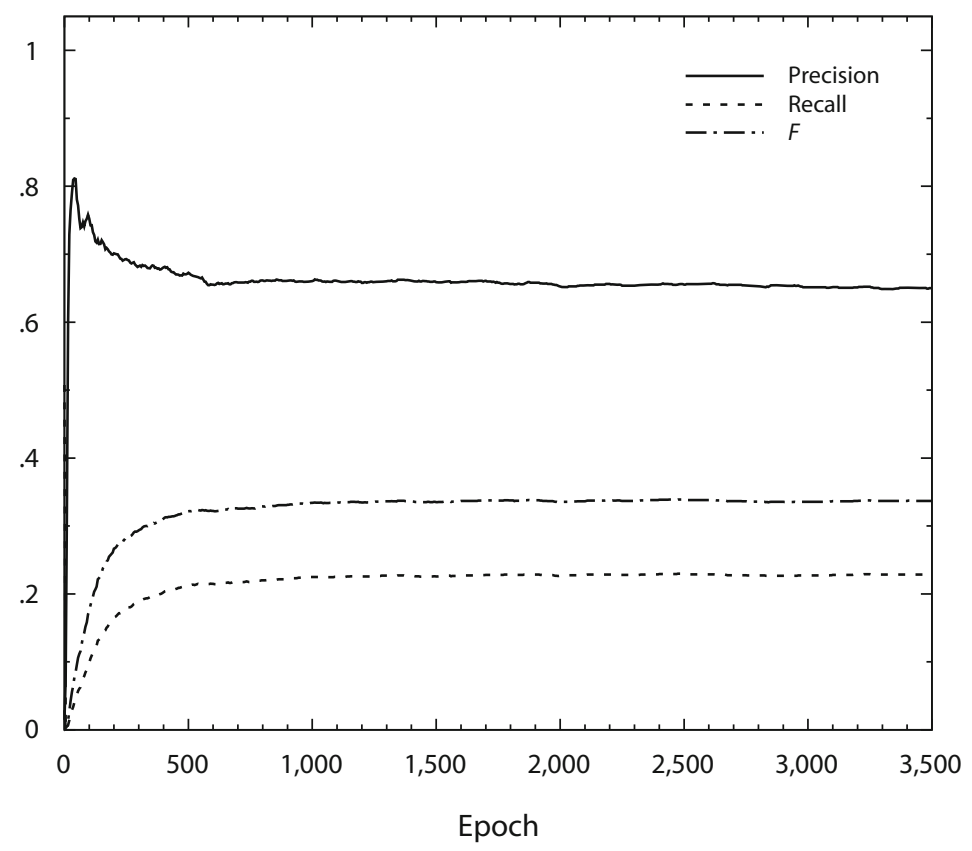

Figure 4. Average precision, recall, and $F$ for 20 testing patterns.

the test items than on the randomized items for precision $[t(9)=31.57, p<.001]$, recall $[t(9)=23.09, p<.001]$, and $F[t(9)=31.12, p<.001]$. Sum-squared error on the test items $(M=37.46, S D=12.94)$ was lower than on the randomized items $(M=57.24, S D=18.16)$ at the outset of training $[t(199)=17.97, p<.001]$. In contrast, at the beginning of training, there was no difference $[t(199)=$ $0.33, p=.742]$ between the average sum-squared error on the test items $(M=1,004.21, S D=21.8)$ and that on the randomized items $(M=1,003.67, S D=23.27)$.

Nearly all of the errors made by the network in its final epoch of training were false negatives, in which a feature was left inactive for a concept that possessed the feature. The total number of false negatives averaged over training sets was $136.1(S D=15.45)$ out of a potential 4,756 false negatives produced if no features are activated, and the average number of false positives was $0.1(S D=0.32)$.

Examining the order in which features are acquired provides some insight into the manner in which the network acquires information about the relationship between co-occurrence vectors and features. For this analysis, we consider a feature to be acquired by the network when its $F$ measure rises above .5. The Appendix contains a table of the first 100 features learned, with the average epoch at which the feature was learned, as well as the number of concepts for which that feature was listed (feature frequency). The features learned earliest are typically indicative of broad categories. For example, $<$ an animal $>,<$ is edible $>,<$ made of metal $>$, and $<$ clothing $>$ are learned after $21.5,33.5,34$, and 58 epochs, respectively. These features group the concepts into four broad categories. Within each of these categories, the network begins to differenti- ate different subcategories and learns features that best achieve this goal, given the current state of knowledge in the network. Within the category of animals, for example, the network learns features that identify large land animals ( $<$ a mammal $>,<$ is large $>,<$ has four legs $>$ ) during epochs 64.5 to 78 . Between epochs 91.5 and 95.5 , the network rapidly learns features that identify the category of fish. During epochs 108.5 to 116.5 , the network begins to produce features that characterize birds ( $<$ has a beak $>$, $<$ a bird $>$, $<$ behavior-flies $>$, $<$ has feathers $>$, and $<$ has wings $>$ ). Within the category of metal objects, the network differentiates between kitchen objects $(<$ found in kitchens $>$, at epoch 80.5), musical instruments ( $<$ a musical instrument $>$, at epoch 90.5), weapons ( $<$ a weapon $>$, at epoch 107.5), and vehicles ( $<$ used for transportation $>$, at epoch 113). The features that are learned initially by the network are typically more general in nature and only roughly categorize the concepts. Table 2 lists the 560th to 580 th features learned by the network. In comparison with the features learned early in training, these features tend either to be specific to a small number of concepts (e.g., $<$ symbol of death $>$, < used for sliding down hills $>$ ) or to describe concepts from a range of categories (e.g., $<$ is pretty $>,<$ found in buildings $>$ ).

It is interesting to note that this learning order is not merely an effect of feature frequency. For example, the feature $<$ is long $>$, which is listed for 81 concepts, is the 33rd feature learned, after 117.5 epochs, whereas the features $<$ a vegetable $>$ and $<$ clothing $>$, the 2 nd and 6 th features learned, are listed for only 28 and 34 concepts, respectively. Rather than learn the most common features first, the network appears to acquire features that best 
Randomized Testing Data

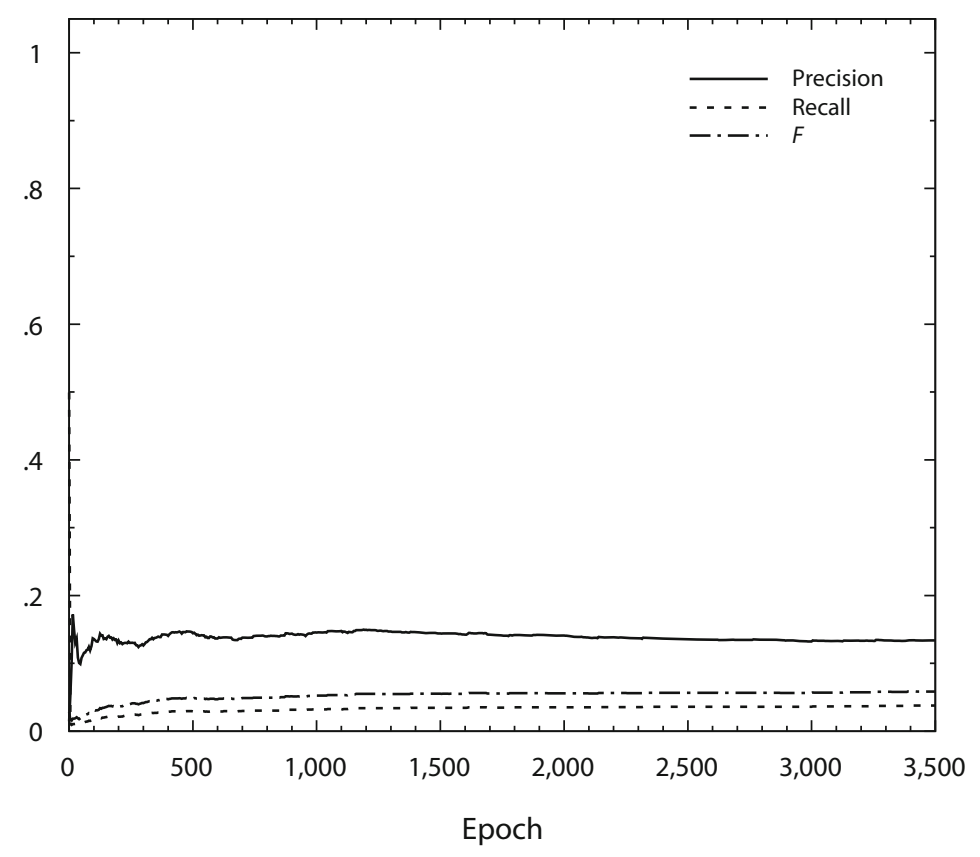

Figure 5. Average precision, recall, and $F$ for 20 randomized testing patterns. Due to the lack of internal structure consistent with the training examples, the network was unable to generalize to these randomized testing patterns.

differentiate the concepts into increasingly specific categories. General features are learned before more itemspecific features not because they are associated with a larger number of concepts, but because they most clearly differentiate concepts during the earliest stages of learning. Furthermore, learning one feature from a coherent group of features describing a category appears to ease the task of learning other features. This is demonstrated by the speed with which the network acquires features describing the categories of fish and, particularly, birds. This manner of learning is similar to that observed in the Rumelhart network of semantic acquisition (Rogers \& McClelland, 2004; Rumelhart, 1990; Rumelhart \& Todd, 1993), in which a hierarchical category structure emerged during learning, with more general features learned earlier than those that are specific to only a few concepts.

\section{Discussion}

Our results provide clear evidence that the network was able to determine a relatively reliable mapping from cooccurrence vectors to featural information. As expected with all machine-learning methods, performance on the training set was superior to that on the test set. However, performance on the test items was above that observed on the randomized test items. This suggests that the network was able to identify regularities in the semantic representations derived entirely from distributional information present in language usage and, to some extent, was able to generalize to novel concepts on the basis of these regularities.

These results are somewhat unsurprising. Earlier, we showed that the representations provided as input to the network are able to reproduce results from semantic and associative priming experiments and can correctly categorize concepts using multidimensional scaling, indicating that the input representations themselves contain a significant amount of information about the meanings of words. The strong performance produced by the cooccurrence vectors in semantic priming and categorization suggests that words with similar meaning have similar co-occurrence vectors. Moreover, words with similar meaning typically correspond to topics that exhibit similar perceptual and functional characteristics, resulting in similar feature vectors. Just as neural networks are able to correctly identify a novel instance of the letter A by generalizing on the basis of similarity to previously experienced instances of the letter, the network used in our experiments was able to identify features of novel concepts by generalizing on the basis of similarity to the concepts on which it was trained. However, to the best of our knowledge, this is the first attempt to develop a mapping from co-occurrence to feature vectors.

Louwerse (2008) argues that embodied representations partially shape the statistical structure of language and that these embodied representations become encoded within this statistical structure. The results above are consistent with this hypothesis. The input representations of concepts were derived entirely from word usage, but the network was nonetheless able to identify reliable cues about the features of the concepts, even when it had not been explicitly trained on a concept. Interestingly, it does not appear that the proportion of concepts that possess a feature is the driving factor behind acquiring knowledge about features. 
Table 2

List of 560th to 580th Features Learned and the Average Number of Training Epochs Required for the Feature to Be Learned

\begin{tabular}{|c|c|c|c|}
\hline \multirow[b]{2}{*}{ Feature } & \multicolumn{2}{|c|}{ Epochs to Learn } & \multirow{2}{*}{$\begin{array}{c}\text { Feature } \\
\text { Frequency }\end{array}$} \\
\hline & $M$ & $S D$ & \\
\hline symbol of death & $1,262.0$ & 543.11 & 2 \\
\hline used for listening to music & $1,264.0$ & 712.83 & 2 \\
\hline used for sliding down hills & $1,288.0$ & 711.58 & 2 \\
\hline is fuzzy/fluffy & $1,293.0$ & 290.96 & 3 \\
\hline behavior - roars & $1,296.5$ & 547.13 & 2 \\
\hline is graceful & $1,297.0$ & 541.48 & 2 \\
\hline is caught in nets & $1,302.5$ & 826.51 & 2 \\
\hline worn for the rain & $1,307.5$ & 874.85 & 3 \\
\hline used on oceans & $1,308.5$ & 832.55 & 2 \\
\hline used for listening & $1,315.0$ & 603.91 & 2 \\
\hline lives in deserts & $1,324.5$ & 537.66 & 6 \\
\hline found in buildings & $1,330.5$ & 695.96 & 3 \\
\hline is pretty & $1,333.5$ & 636.94 & 7 \\
\hline found on walls & $1,355.5$ & 569.35 & 7 \\
\hline used on snow & $1,359.0$ & 750.87 & 3 \\
\hline used by hanging from ceilings & $1,361.0$ & 892.83 & 2 \\
\hline has bristles & $1,388.5$ & 790.57 & 3 \\
\hline has a drain plug & $1,459.5$ & $1,113.13$ & 2 \\
\hline used by hanging things on & $1,463.0$ & 329.34 & 3 \\
\hline has a cap & $1,464.0$ & 944.22 & 2 \\
\hline
\end{tabular}

Note-Feature frequency, number of concepts for which subjects produced the feature.

Rather, the network first identifies features that are best able to divide the concepts into broad categories, learning more specific features in the later stages of training. Category coherence also seems to play a role in learning. Support for this comes from the observation that coherent groups of features describing a category were often learned in quick succession, whereas features describing concepts from a variety of categories are typically learned later than are those that are more distinct to a specific category. These observations are similar to those of Rogers and McClelland (2004), who used a small artificial corpus of concepts to investigate the nature of semantic knowledge acquisition. In the present work, we have extended these previous results to show that the same holds true for representations derived from language usage and feature vectors produced by subjects.

One obvious shortcoming of the present work is the simple architecture that was used-namely, a three-layer feed-forward network. Ideally, given a set of features that describe a concept, the network should be able to activate its co-occurrence-based semantic representation. However, the network operates in only a single direction, allowing activation at the input units to propagate through the network to the output units, and is therefore unable to access co-occurrence information when it is provided a set of features.

This simple architecture introduces an additional difficulty that is more relevant to the network's performance. It has been shown that feature correlation plays an important role in concept acquisition and later access to these concepts (McRae et al., 1999; McRae et al., 1997; Rogers \& McClelland, 2004). For example, the category of birds is described by the features $<$ a_bird $>$, $<$ has_wings $>$, $<$ has_feathers $>$, and $<$ has_a_beak $>$. These features form a coherent group and, given a concept that possesses any three of these features, it is likely that the concept would also possess the fourth. In the present model, there is no means by which information can be passed between the feature units: There are no recurrent connections between the hidden and output layers, nor any type of "semantic cleanup" units of the form that are included in many connectionist models to introduce attractor dynamics (Hinton \& Shallice, 1991; Plaut, 1995; Plaut \& Shallice, 1993). Thus, unless information about feature correlations is explicitly represented in the input vectors, the network is unable to take full advantage of knowledge of these coherent groups of features. Including recurrent connections between the feature units may allow the network to exploit correlations between features as a complement to the general information provided by the semantic vectors used as input to the network. Given a concept that activates several features from a coherent feature group, the feature correlation information provided by recurrent connections may allow the network to activate other features from that group that are represented with only low salience in the input vector and would otherwise not be activated.

One likely difficulty with this type of model that was not explored concerns how features are generalized to novel concepts. Since the network is able to generalize on the basis of similarity between co-occurrence vectors and is also able to account for associative priming in experiments such as Ferrand and New's (2004), it is reasonable to believe that the network will incorrectly attribute features from one concept to other concepts that are associatively related but share little in terms of featural similarity. For example, meow and cat are closely related through association. Will the network incorrectly generate features such as $<$ has fur $>$ and $<$ has a tail $>$ to the concept meow? This is a question that requires considerable analysis to answer and, for the time being, is acknowledged as a potential and likely difficulty that we relegate to the realm of future work.

The work in this article has taken a step toward addressing a shortcoming of co-occurrence models, but other difficulties remain. In this model, co-occurrence vectors were mapped onto a set of representational units that each corresponded to a single feature. Each feature unit was a localist representation that indicated only the presence or absence of a single feature, and these features were derived from norms produced by human subjects. The question of how the model could develop such features on its own, in the same manner as that by which it had acquired general semantic knowledge - directly from word co-occurrences in text-is difficult. The model would not only need to develop a distributed representation for, for example, each different species of bird, but would also need to develop a more general concept of bird and associate this representation with those of each different species. In addition, the model would need to develop a concept of wing and associate this concept with the various representations of birds, both at the individual-species and more general levels. This concept would, in turn, need to be associated with representations of bones and feath- 
ers, and its other component parts. Landauer and Dumais (1997) suggested that this regression ends only when the concepts become grounded in their perceptual representations. This indicates that models derived entirely from word co-occurrence are necessarily impoverished unless the representations can be grounded in some set of perceptual primitives that provide the substrate for the various modes of representation in the brain. Again, recurrent connections may be able to provide a partial solution to this problem by allowing representations of features to be associated with the more general semantic information provided by the co-occurrence vectors. The feature $<$ has_wings $>$, for example, could be related to the cooccurrence representation of the concept wing.

The present study demonstrated that it is possible to extract features from co-occurrence-based representations of semantic memory. This is a step toward addressing a commonly raised and difficult problem for co-occurrence models: The representations produced by co-occurrence models are not grounded. This criticism was brought to light by work in the field of grounded (or embodied) cognition, in which the symbol-grounding problem was introduced as a problem for most models of memory. In the theory of grounded cognition, knowledge is acquired by integrating multimodal representations of experiences with the world, body, and mind into a common representational memory system. Later recall of this knowledge occurs through a process of simulation, in which the brain reproduces the multimodal representations that were captured during knowledge acquisition. Simulation is assumed to be a core method of computation in the brain, operating on a representational system that is shared between processing systems. In this theory, cognition is closely tied to the perceptual and motor systems, as well as to introspective and emotional states. Taken as a definition of semantic memory, this reaches beyond the more basic definition of a general storehouse of knowledge about words and the world and the relationships between them-essentially, a dictionary, thesaurus, and encyclopedia rolled into one (McNamara \& Holbrook, 2003).

A problem closely related to the symbol-grounding problem is that the richness of human experience cannot be used to inform a computational model of semantic knowledge. Our experience with the world occurs in a variety of media, such as visual and other forms of perceptual input, but computational models of semantic memory are limited to purely linguistic input. Due to this inability to integrate nonlinguistic input, representations constructed solely from co-occurrence data must be impoverished and, thus, are unable to fully represent the complexity of human semantics.

These problems have been used to suggest that cooccurrence models exist in opposition to grounded cognition, but we believe quite the opposite. Consistent with the theory of grounded cognition, linguistic input forms only a single component of the input to the semantic system. Burgess $(1998,2000)$ argued that the co-occurrence representations derived by the HAL model are grounded because each component of a word's semantic vector measures the strength of association between that word and some other word from the corpus. Here, we have shown that information about featural characteristics can be represented in a latent manner throughout a vector representation and can be used to identify features of a concept from co-occurrence representations in which the axes are arbitrary and have no clear interpretation. This offers a more direct form of symbol grounding. Unfortunately, such simple computational models lack the large array of processing mechanisms that exist in the brain and that are posited to participate in the computational mechanism of simulation that is central to grounded cognition. In our model, we can interpret each localist feature node as a great simplification of the rich multimodal representations on which the simulation process operates. Under this interpretation, we feel that co-occurrence is consistent with the principles of grounded cognition.

This is not to say that we believe the model presented here is fully grounded. As we mentioned above, a truly grounded model must be able to represent the features themselves in a distributed and multimodal manner across the various processing systems in the brain. In this article, we have demonstrated that representations derived from language usage contain information related to the features of concepts and that this information can be exploited in a simple neural network architecture. Our results support the work of Louwerse (2008) by showing that language partially encodes information about embodied representations. We are careful not to make the claim that vectors produced by methods that are based on word co-occurrence are fully grounded representations, but we are confident that the representations produced by such methods are at least partially grounded and provide a practical and psychologically valid alternative to representations derived from feature norms collected from human subjects.

\section{AUTHOR NOTE}

This research was supported by grants from NSERC to L.B. and R.C. We are grateful to Mike Jones for comments on an early version of this article. Correspondence concerning this article should be addressed to L. Buchanan, Department of Psychology, University of Windsor, Windsor, ON, N9B 3P4 Canada (e-mail: buchanan@uwindsor.ca).

\section{REFERENCES}

Andrews, M., Vigliocco, G., \& Vinson, D. (2005). Integrating attributional and distributional information in a probabilistic model of meaning representation. In T. Honkela, V. Könönen, M. Pöllä, \& O. Simula (Eds.), Proceedings of the International and Interdisciplinary Conference on Adaptive Knowledge Representation and Reasoning (pp. 1525). Espoo, Finland

Andrews, M., Vigliocco, G., \& Vinson, D. (2007). Evaluating the contribution of intra-linguistic and extra-linguistic data to the structure of human semantic representations. In D. S. McNamara \& J. G. Trafton (Eds.), Proceedings of the 29th Annual Conference of the Cognitive Science Society (pp. 767-772). Austin, TX: Cognitive Science Society.

Andrews, M., Vigliocco, G., \& Vinson, D. (2009). Integrating experiential and distributional data to learn semantic representations. Psychological Review, 116, 463-498.

Barsalou, L. W. (2008). Grounded cognition. Annual Review of Psychology, 59, 617-645.

BuRGESS, C. (1998). From simple associations to the building blocks of language: Modeling meaning in memory with the HAL model. $B e-$ havior Research Methods, Instruments, \& Computers, 30, 188-198.

Burgess, C. (2000). Theory and operational definitions in computa- 
tional memory models: A response to Glenberg and Robertson. Journal of Memory \& Language, 43, 402-408.

BURGESS, C. (2001). Representing and resolving semantic ambiguity: A contribution from high-dimensional memory modeling. In D. S. Gorfein (Ed.), On the consequences of meaning selection: Perspectives on resolving lexical ambiguity (pp. 233-260). Washington, DC: American Psychological Association

Burgess, C., \& Lund, K. (1997a). Modelling parsing constraints with high-dimensional context space. Language \& Cognitive Processes, 12, 177-210.

Burgess, C., \& Lund, K. (1997b). Representing abstract words and emotional connotation in a high-dimensional memory space. In M. G. Shafto \& P. Langley (Eds.), Proceedings of the 19th Annual Conference of the Cognitive Science Society (pp. 61-66). Mahwah, NJ: Erlbaum.

Burgess, C., \& Lund, K. (2000). The dynamics of meaning in memory. In E. Dietrich \& A. B. Markman (Eds.), Cognitive dynamics (pp. 117156). Mahwah, NJ: Erlbaum.

Collins, A. M., \& LofTus, E. F. (1975). A spreading-activation theory of semantic processing. Psychological Review, 82, 407-428.

Collins, A. M., \& Quillian, M. R. (1969). Retrieval time from semantic memory. Journal of Verbal Learning \& Verbal Behavior, 8 , 240-247.

DAMERAU, F. (1993). Generating and evaluating domain-oriented multiword terms from texts. Information Processing \& Management, 29, 433-448.

DURDA, K., \& BUCHANAN, L. (2008). WINDSORS: Windsor improved norms of distance and similarity of representations of semantics. Behavior Research Methods, 40, 705-712.

Elman, J. L. (1990). Finding structure in time. Cognitive Science, 14, 179-211.

Fellbaum, C. (1998). WordNet: An electronic lexical database. Cambridge, MA: MIT Press.

FERRAND, L., \& New, B. (2004). Semantic and associative priming in the mental lexicon. In P. Bonin (Ed.), Mental lexicon: Some words to talk about words (pp. 25-43). Hauppauge, NY: Nova Science.

French, R. M., \& Labiouse, C. (2002). Four problems with extracting human semantics from large text corpora. In W. D. Gray \& C. D. Schunn (Eds.), Proceedings of the 24th Annual Conference of the Cognitive Science Society (pp. 316-322). Mahwah, NJ: Erlbaum

GLENBERG, A. M. (1997). What memory is for. Behavioral \& Brain Sciences, 20, $1-55$.

Glenberg, A. M., \& Robertson, D. A. (2000). Symbol grounding and meaning: A comparison of high-dimensional and embodied theories of meaning. Journal of Memory \& Language, 43, 379-401

Griffiths, T. L., \& STEYVERS, M. (2002). A probabilistic approach to semantic representation. In W. D. Gray \& C. D. Schunn (Eds.), Proceedings of the 24th Annual Conference of the Cognitive Science Society (pp. 381-386). Mahwah, NJ: Erlbaum.

Griffiths, T. L., \& Steyvers, M. (2003). Prediction and semantic association. In S. Becker, S. Thrun, \& K. Obermayer (Eds.), Advances in neural information processing systems 15 (pp. 11-18). Cambridge, MA: MIT Press.

Griffiths, T. L., Steyvers, M., \& Tenenbaum, J. B. (2007). Topics in semantic representation. Psychological Review, 114, 211-244.

Harm, M. W., \& SeidenberG, M. S. (2004). Computing the meanings of words in reading: Cooperative division of labor between visual and phonological processes. Psychological Review, 111, 662-720.

Hinton, G. E., McClelland, J. L., \& Rumelhart, D. E. (1986). Distributed representations. In D. E. Rumelhart, J. L. McClelland, \& the PDP Research Group (Eds.), Parallel distributed processing: Explorations in the microstructure of cognition: Vol. 1. Foundations (pp. 77109). Cambridge, MA: MIT Press.

Hinton, G. E., \& Shallice, T. (1991). Lesioning an attractor network: Investigations of acquired dyslexia. Psychological Review, 98, 74-95.

Holland, J. H. (1992). Adaptation in natural and artificial systems. Cambridge, MA: MIT Press.

Howell, S. R., Jankowicz, D., \& Becker, S. (2005). A model of grounded language acquisition: Sensorimotor features improve lexical and grammatical learning. Journal of Memory \& Language, $\mathbf{5 3}$, 258-276.

Jones, M. N., Kintsch, W., \& Mewhort, D. J. K. (2006). High- dimensional semantic space accounts of priming. Journal of Memory \& Language, 55, 534-552.

JONES, M. N., \& MEWHORT, D. J. K. (2007). Representing word meaning and order information in a composite holographic lexicon. Psychological Review, 114, 1-37.

Kullback, S., \& Leibler, R. A. (1951). On information and sufficiency. Annals of Mathematical Statistics, 22, 79-86.

Landauer, T. K., \& Dumais, S. T. (1997). A solution to Plato's problem: The latent semantic analysis theory of acquisition, induction, and representation of knowledge. Psychological Review, 104, 211-240.

Lemaire, B., \& Denhiére, G. (2004). Incremental construction of an associative network from a corpus. In K. D. Forbus, D. Gentner, \& T. Regier (Eds.), Proceedings of the 26th Annual Meeting of the Cognitive Science Society (pp. 825-830). Mahwah, NJ: Erlbaum.

Louwerse, M. M. (2007). Symbolic or embodied representations: A case for symbol interdependency. In T. K. Landauer, D. S. McNamara, S. Dennis, \& W. Kintsch (Eds.), Handbook of latent semantic analysis (pp. 107-120). Mahwah, NJ: Erlbaum.

LOUWERSE, M. M. (2008). Embodied relations are encoded in language. Psychonomic Bulletin \& Review, 15, 838-844.

Louwerse, M. M., Cai, Z., Hu, X., Ventura, M., \& Jeuniaux, P. (2006). Cognitively inspired natural-language based knowledge representations: Further explorations of latent semantic analysis. International Journal of Artificial Intelligence Tools, 15, 1021-1040.

Lund, K., \& Burgess, C. (1996). Producing high-dimensional semantic spaces from lexical co-occurrence. Behavior Research Methods, Instruments, \& Computers, 28, 203-208.

Martin, A., \& ChaO, L. L. (2001). Semantic memory and the brain: Structure and processes. Current Opinion in Neurobiology, 11, 194-201.

McNamara, T. P., \& Holbrook, J. B. (2003). Semantic memory and priming. In I. B. Weiner (Series Ed.) \& A. F. Healy \& R. W. Proctor (Vol. Eds.), Handbook of psychology: Vol. 4. Experimental psychology (pp. 447-474). Hoboken, NJ: Wiley.

McRae, K., Cree, G. S., Seidenberg, M. S., \& McNorgan, C. (2005). Semantic feature production norms for a large set of living and nonliving things. Behavior Research Methods, 37, 547-559.

McRae, K., Cree, G. S., Westmacott, R., \& DE Sa, V. R. (1999). Further evidence for feature correlations in semantic memory. Canadian Journal of Experimental Psychology, 53, 360-373.

McRae, K., DE SA, V. R., \& SeIdenberg, M. S. (1997). On the nature and scope of featural representations of word meaning. Journal of Experimental Psychology: General, 126, 99-130.

Pexman, P. M., Holyk, G. G., \& MonfiLs, M.-H. (2003). Number-offeatures effects and semantic processing. Memory \& Cognition, 31, 842-855.

Plaut, D. C. (1995). Semantic and associative priming in a distributed attractor network. In J. D. Moore \& J. F. Lehman (Eds.), Proceedings of the 17th Annual Conference of the Cognitive Science Society (pp. 37-42). Mahwah, NJ: Erlbaum.

Plaut, D. C., \& Bоoth, J. R. (2000). Individual and developmental differences in semantic priming: Empirical and computational support for a single-mechanism account of lexical processing. Psychological Review, 107, 786-823.

Plaut, D. C., \& Shallice, T. (1993). Deep dyslexia: A case study of connectionist neuropsychology. Cognitive Neuropsychology, 10, 377-500.

RIORDAN, B., \& JONES, M. N. (in press). Redundancy in perceptual and linguistic experience: Comparing feature-based and distributional models of semantic representation. Topics in Cognitive Science.

Rodd, J. M., Gaskell, M. G., \& Marslen-Wilson, W. D. (2004). Modelling the effects of semantic ambiguity in word recognition. Cognitive Science, 28, 89-104.

Rogers, T. T., \& McClelland, J. L. (2004). Semantic cognition: A parallel distributed processing approach. Cambridge, MA: MIT Press.

Rohde, D. L. T., Gonnerman, L. M., \& Plaut, D. C. (2004). An improved method for deriving word meaning from lexical co-occurrence. Unpublished manuscript.

RuMELHART, D. E. (1990). Brain style computation: Learning and generalization. In S. F. Zornetzer, J. L. Davis, \& C. Lau (Eds.), An introduction to neural and electronic networks (pp. 405-420). San Diego: Academic Press.

Rumelhart, D. E., Hinton, G. E., \& Williams, R. J. (1986). Learn- 
ing internal representations by error propagation. In D. E. Rumelhart, J. L. McClelland, \& the PDP Research Group (Eds.), Parallel distributed processing: Explorations in the microstructure of cognition. Vol. 1: Foundations (pp. 318-362). Cambridge, MA: MIT Press.

RumelharT, D. E., \& TodD, P. M. (1993). Learning and connectionist representations. In D. E. Meyer \& S. Kornblum (Eds.), Attention and performance XIV: Synergies in experimental psychology, artificial intelligence, and cognitive neuroscience (pp. 3-30). Cambridge, MA: MIT Press.

SAFFran, E. M. (2000). The organization of semantic memory: In support of a distributed model. Brain \& Language, 71, 204-212.

ScHÜTZe, H. (1992). Dimensions of meaning. In Proceedings of the 1992 ACM/IEEE Conference on Supercomputing (pp. 787-796). Los Alamitos, CA: IEEE Computer Society Press.

Schütze, H. (1998). Automatic word sense discrimination. Computational Linguistics, 24, 97-124.

Shaoul, C., \& Westbury, C. (2006). Word frequency effects in highdimensional co-occurrence models: A new approach. Behavior Research Methods, 38, 190-195.

Shaoul, C., \& Westbury, C. (2008a). Performance of HAL-like word space models on semantic clustering. In M. Baroni \& A. Lenci (Eds.), ESSLLI lexical semantics workshop: Bridging the gap between semantic theory and computational simulations (pp. 42-46). Hamburg: ESSLLI.

Shaoul, C., \& Westbury, C. (2008b). A USENET corpus (2005-2008). Edmonton, Alberta: University of Alberta. Retrieved from www .psych.ualberta.ca/ westburylab/downloads/usenetcorpus.download .html.

Sitnikova, T., West, W. C., Kuperberg, G. R., \& Holcomb, P. J. (2006). The neural organization of semantic memory: Electrophysiological activity suggests feature-based segregation. Biological Psychology, 71, 326-340.

Sloman, S. A. (1998). Categorical inference is not a tree: The myth of inheritance hierarchies. Cognitive Psychology, 35, 1-33.

Steyvers, M., \& Tenenbaum, J. B. (2005). The large-scale structure of semantic networks: Statistical analyses and a model of semantic growth. Cognitive Science, 29, 41-78.

Vigliocco, G., Warren, J., Siri, S., Arciuli, J., Scott, S., \& Wise, R. (2006). The role of semantics and grammatical class in the neural representation of words. Cerebral Cortex, 16, 1790-1796. 
APPENDIX

List of the First 100 Features Learned by the Network, With Average Number of Epochs Required for Learning

\begin{tabular}{|c|c|c|c|c|c|c|c|}
\hline \multirow[b]{2}{*}{ Feature } & \multicolumn{2}{|c|}{$\begin{array}{l}\text { Epochs } \\
\text { to Learn }\end{array}$} & \multirow{2}{*}{$\begin{array}{c}\text { Feature } \\
\text { Frequency }\end{array}$} & \multirow[b]{2}{*}{ Feature } & \multicolumn{2}{|c|}{$\begin{array}{l}\text { Epochs } \\
\text { to Learn }\end{array}$} & \multirow{2}{*}{$\begin{array}{c}\text { Feature } \\
\text { Frequency }\end{array}$} \\
\hline & $M$ & $S D$ & & & $M$ & $S D$ & \\
\hline an animal & 21.5 & 3.37 & 99 & is fast & 157.0 & 16.87 & 33 \\
\hline a vegetable & 33.0 & 5.37 & 28 & used for cooking & 160.0 & 35.75 & 13 \\
\hline is edible & 33.5 & 4.74 & 78 & worn by women & 166.0 & 76.80 & 18 \\
\hline made of metal & 34.0 & 3.16 & 133 & is soft & 177.0 & 25.41 & 27 \\
\hline clothing & 58.0 & 15.13 & 34 & is comfortable & 179.5 & 35.39 & 15 \\
\hline grows in gardens & 61.5 & 14.54 & 18 & has leaves & 183.0 & 55.04 & 15 \\
\hline a fruit & 62.5 & 24.41 & 29 & has fur & 183.0 & 41.38 & 22 \\
\hline a mammal & 64.5 & 9.56 & 42 & has skin & 197.5 & 72.81 & 16 \\
\hline is large & 72.5 & 9.20 & 106 & is yellow & 198.5 & 48.54 & 27 \\
\hline is small & 73.5 & 6.69 & 121 & is red & 204.0 & 44.15 & 26 \\
\hline eaten by cooking & 74.5 & 16.06 & 15 & is white & 208.5 & 22.12 & 51 \\
\hline has 4 legs & 78.0 & 10.33 & 49 & is black & 215.0 & 34.24 & 50 \\
\hline tastes sweet & 78.0 & 20.30 & 24 & lives on farms & 220.0 & 85.63 & 12 \\
\hline found in kitchens & 80.5 & 18.33 & 32 & has strings & 220.5 & 128.12 & 9 \\
\hline is green & 80.5 & 13.01 & 47 & a tool & 222.0 & 51.87 & 18 \\
\hline different colours & 83.0 & 13.37 & 58 & furniture & 229.0 & 94.22 & 11 \\
\hline made of wood & 89.5 & 11.17 & 79 & used for storage & 230.5 & 55.25 & 18 \\
\hline a musical instrument & 90.5 & 35.31 & 18 & is sharp & 231.5 & 68.23 & 18 \\
\hline behavior - swims & 91.5 & 14.73 & 33 & is crunchy & 239.0 & 80.34 & 11 \\
\hline lives in water & 94.0 & 14.68 & 32 & used for passengers & 244.5 & 63.62 & 9 \\
\hline has a tail & 95.5 & 16.24 & 39 & an insect & 247.0 & 112.40 & 12 \\
\hline eaten in salads & 101.0 & 29.51 & 13 & a utensil & 250.5 & 51.34 & 12 \\
\hline is nutritious & 101.5 & 32.92 & 15 & has doors & 254.5 & 54.13 & 15 \\
\hline grows on trees & 107.0 & 24.29 & 19 & is heavy & 256.5 & 24.04 & 27 \\
\hline a weapon & 107.5 & 24.52 & 29 & is electrical & 256.5 & 65.19 & 22 \\
\hline has a handle & 107.5 & 14.19 & 42 & made of leather & 259.0 & 64.37 & 13 \\
\hline has a beak & 108.5 & 26.67 & 38 & used in bands & 262.5 & 159.03 & 9 \\
\hline a bird & 110.0 & 16.83 & 39 & used for juice & 263.5 & 119.86 & 7 \\
\hline used for transportation & 113.0 & 19.61 & 33 & comes in pairs & 263.5 & 75.72 & 7 \\
\hline behavior - flies & 113.5 & 17.49 & 46 & a fish & 265.0 & 119.28 & 16 \\
\hline has feathers & 115.5 & 26.50 & 38 & is expensive & 266.5 & 38.37 & 22 \\
\hline has wings & 116.5 & 22.98 & 44 & tastes sour & 268.0 & 195.44 & 8 \\
\hline is long & 117.5 & 10.61 & 81 & is furry & 269.0 & 69.79 & 20 \\
\hline behavior - eats & 117.5 & 20.03 & 39 & has teeth & 269.5 & 29.48 & 14 \\
\hline tastes good & 119.0 & 13.70 & 33 & has eyes & 270.5 & 98.16 & 16 \\
\hline is juicy & 120.0 & 22.48 & 21 & a food & 272.0 & 57.55 & 9 \\
\hline is round & 121.5 & 14.54 & 76 & has fins & 273.0 & 103.63 & 11 \\
\hline has legs & 123.0 & 14.18 & 44 & used for war & 281.0 & 88.37 & 12 \\
\hline hunted by people & 124.0 & 15.78 & 27 & used for cutting & 283.0 & 92.68 & 9 \\
\hline made of plastic & 127.0 & 15.13 & 51 & is thin & 284.5 & 59.46 & 16 \\
\hline has seeds & 133.5 & 19.16 & 21 & a vehicle & 289.0 & 103.41 & 10 \\
\hline used for killing & 134.0 & 28.26 & 20 & different sizes & 291.5 & 58.55 & 19 \\
\hline inanimate behavior - & 136.5 & 59.44 & 13 & eaten in pies & 295.0 & 144.05 & 5 \\
\hline produces music & & & & has pockets & 298.0 & 181.63 & 6 \\
\hline is brown & 138.5 & 20.42 & 54 & has gills & 299.0 & 149.35 & 9 \\
\hline is dangerous & 139.0 & 24.01 & 37 & an appliance & 301.5 & 75.17 & 10 \\
\hline behavior - lays eggs & 145.0 & 24.72 & 32 & has peel & 302.5 & 105.63 & 6 \\
\hline made of material & 146.0 & 37.55 & 18 & has scales & 304.0 & 110.67 & 10 \\
\hline is loud & 152.5 & 23.83 & 34 & made of cotton & 304.5 & 133.51 & 12 \\
\hline worn for warmth & 155.5 & 25.54 & 15 & is hard & 306.0 & 71.87 & 22 \\
\hline has wheels & 157.0 & 59.17 & 23 & & & & \\
\hline
\end{tabular}

Note-Feature frequency, number of concepts for which at least 1 subject produced the feature.

Manuscript received January 12, 2009; 